\title{
Normal projections in Krein spaces
}

\author{
Alejandra Maestripieri*and Francisco Martínez Pería ${ }^{\dagger}$
}

\begin{abstract}
Given a complex Krein space $\mathcal{H}$ with fundamental symmetry $J$, the aim of this note is to characterize the set of $J$-normal projections

$$
\mathcal{Q}=\left\{Q \in L(\mathcal{H}): Q^{2}=Q \text { and } Q^{\#} Q=Q Q^{\#}\right\} .
$$

The ranges of the projections in $\mathcal{Q}$ are exactly those subspaces of $\mathcal{H}$ which are pseudo-regular. For a fixed pseudo-regular subspace $\mathcal{S}$, there are infinitely many $J$-normal projections onto it, unless $\mathcal{S}$ is regular. Therefore, most of the material herein is devoted to parametrizing the set of $J$-normal projections onto a fixed pseudo-regular subspace $\mathcal{S}$.
\end{abstract}

AMS Math. Subject Classification: Primary 46C20, 47B50; Secondary 47A62. Keywords: Normal operator, projection, Krein space.

\section{Introduction}

It is well-known that a (linear, bounded) projection $Q$, acting on a Hilbert space $\mathcal{H}$, is normal $\left(Q Q^{*}=Q^{*} Q\right)$ if and only if it is selfadjoint $\left(Q=Q^{*}\right)$. Therefore, there is a one-to-one correspondence between the closed subspaces of $\mathcal{H}$ and the normal projections acting on $\mathcal{H}$.

On the other hand, if $\mathcal{K}$ is a Krein space with fundamental symmetry $J$, it is easy to find $J$-normal projections which are not $J$-selfadjoint (see Example 1 in Section 3). For a fixed Krein space $\mathcal{K}$ with fundamental symmetry $J$, the purpose of this work is to describe those projections acting on $\mathcal{K}$ which are $J$-normal, i.e. those $Q=Q^{2} \in L(\mathcal{K})$ satisfying

$$
Q Q^{\#}=Q^{\#} Q,
$$

where $Q^{\#}$ is the $J$-adjoint of $Q$.

If $Q$ is $J$-normal, observe that $E=Q Q^{\#}$ is a $J$-selfadjoint projection whose range, hereafter denoted by $R(E)$, is contained in $R(Q)$. Thus, $R(Q)$ contains a regular subspace of $\mathcal{K}$. On the other hand, $P=Q\left(I-Q^{\#}\right)$ is a projection with $R(P)=R(Q) \cap R(Q)^{[\perp]}=R(Q)^{\circ}$, i.e. $R(P)$ is the isotropic part of $R(Q)$.

\footnotetext{
*A. Maestripieri was partially supported by PICT FONCYT 808.

${ }^{\dagger}$ F. Martínez Pería was partially supported by PICT FONCYT 808 and PIP CONICET 0435.
} 
Also, since $E P=P E=0$ it follows that $Q=E+P$ and

$$
R(Q)=R(E)[\dot{+}] R(P)=R(E)[\dot{+}] R(Q)^{\circ},
$$

that is, $R(Q)$ is a pseudo-regular subspace of $\mathcal{K}$, see [9] for the terminology. Conversely, it will be shown that every pseudo-regular subspace of $\mathcal{K}$ admits a $J$-normal projection onto it. However, it is not hard to prove that a pseudoregular subspace may admit infinitely many $J$-normal projections onto it (see Example 2 in Section 4).

The importance of pseudo-regular subspaces lies in the fact that they enable to generalize some Pontryagin spaces arguments to general Krein spaces, see [9]. They have also been used as a technical tool for the study of spectral functions (and distributions) for particular classes of operators in Krein spaces $[10,11,13,14]$ and to extend the Beurling-Lax theorem for shifts in indefinite metric spaces $[4,5]$.

Along this work, different characterizations of $J$-normal projections will be developed. Furthermore, for a fixed pseudo-regular subspace $\mathcal{S}$, we will present a parametrization for the set of $J$-normal projections onto $\mathcal{S}$.

In the next section we introduce the basic notations and terminology used in the paper. Section 3 is devoted to describe $J$-normal projections. In particular, it is shown that every $J$-normal projection $Q$ admits a unique decomposition $Q=E+P$ where $E$ is $J$-selfadjoint and $P$ is a $J$-normal projection with $J$ neutral range. Then, the main consequences of this decomposition are discussed.

In Section 4 it is shown that a (closed) subspace $\mathcal{S}$ is the range of a $J$-normal projection if and only if it is pseudo-regular, i.e. if $\mathcal{S}+\mathcal{S}^{[\perp]}$ is closed. Then, although there is not a unique $J$-normal projection onto an arbitrary pseudoregular subspace $\mathcal{S}$, a formula for a particular $J$-normal projection onto $\mathcal{S}$ is presented (depending only on the fundamental symmetry $J$ and the orthogonal projections onto $\mathcal{S}$ and $\mathcal{S}^{\circ}$ ).

Section 5 deals with $J$-normal projections onto $J$-neutral subspaces. It will be shown that there are infinitely many $J$-normal projections onto a prescribed $J$-neutral subspace (and their nullspaces can be arbitrarily close). Then, for a fixed $J$-neutral subspace $\mathcal{N}$, a parametrization for the set of $J$-normal projections onto $\mathcal{N}$ is presented.

Finally, the aim of Section 6 is to present an explicit description of the set of $J$-normal projections onto a pseudo-regular subspace $\mathcal{S}$. First, it is shown that this set can be decomposed in a disjoint union of decks. Then, considering the projections as block-operator matrices according to an appropriate orthogonal decomposition, each deck is parametrized.

\section{Preliminaries}

Notation and terminology Along this work $\mathcal{H}$ denotes a complex (separable) Hilbert space. If $\mathcal{K}$ is another Hilbert space then $L(\mathcal{H}, \mathcal{K})$ is the algebra of bounded linear operators from $\mathcal{H}$ into $\mathcal{K}$ and $L(\mathcal{H})=L(\mathcal{H}, \mathcal{H})$. The 
group of linear invertible operators acting on $\mathcal{H}$ is denoted by $G L(\mathcal{H})$. Also, $L(\mathcal{H})^{+}$denotes the cone of positive semidefinite operators acting on $\mathcal{H}$ and $G L(\mathcal{H})^{+}=G L(\mathcal{H}) \cap L(\mathcal{H})^{+}$.

If $T \in L(\mathcal{H}, \mathcal{K})$ then $T^{*} \in L(\mathcal{K}, \mathcal{H})$ denotes the adjoint operator of $T, R(T)$ stands for its range and $N(T)$ for its nullspace.

Given two closed subspaces $\mathcal{S}$ and $\mathcal{T}$ of a Hilbert space $\mathcal{H}, \mathcal{S}+\mathcal{T}$ denotes the direct sum of them. On the other hand, $\mathcal{S} \oplus \mathcal{T}$ stands for their (direct) orthogonal sum and $\mathcal{S} \ominus \mathcal{T}:=\mathcal{S} \cap(\mathcal{S} \cap \mathcal{T})^{\perp}$. If $\mathcal{H}=\mathcal{S}+\mathcal{T}$, there exists a (unique) bounded projection with range $\mathcal{S}$ and nullspace $\mathcal{T}$. Hereafter, it is denoted by $P_{\mathcal{S} / / \mathcal{T}}$. If $P_{\mathcal{S}}$ and $P_{\mathcal{T}}$ stand for the orthogonal projections onto $\mathcal{S}$ and $\mathcal{T}$, respectively, $P_{\mathcal{S} / / \mathcal{T}}$ can be represented as:

$$
P_{\mathcal{S} / / \mathcal{T}}=P_{\mathcal{S}}\left(P_{\mathcal{S}}+P_{\mathcal{T}}\right)^{-1},
$$

see [2, Lemma 3.1].

Given two closed subspaces $\mathcal{S}$ and $\mathcal{T}$ of a Hilbert space $\mathcal{H}$, the cosine of the Friedrichs angle between $\mathcal{S}$ and $\mathcal{T}$ is defined by

$$
c(\mathcal{S}, \mathcal{T})=\sup \{|\langle x, y\rangle|: x \in \mathcal{S} \ominus \mathcal{T},\|x\|=1, y \in \mathcal{T} \ominus \mathcal{S},\|y\|=1\} .
$$

It is well known that

$$
c(\mathcal{S}, \mathcal{T})<1 \Leftrightarrow \mathcal{S}+\mathcal{T} \text { is closed } \Leftrightarrow c\left(\mathcal{S}^{\perp}, \mathcal{T}^{\perp}\right)<1 .
$$

Furthermore, if $P_{\mathcal{S}}$ and $P_{\mathcal{T}}$ are the orthogonal projections onto $\mathcal{S}$ and $\mathcal{T}$, respectively, then $c(\mathcal{S}, \mathcal{T})<1$ if and only if $\left(I-P_{\mathcal{S}}\right) P_{\mathcal{T}}$ has closed range.

On the other hand, the Dixmier (or minimal) angle between $\mathcal{S}$ and $\mathcal{T}$ is defined by

$$
c_{0}(\mathcal{S}, \mathcal{T})=\sup \{|\langle x, y\rangle|: x \in \mathcal{S},\|x\|=1, y \in \mathcal{T},\|y\|=1\} .
$$

It is clear that $c(\mathcal{S}, \mathcal{T}) \leq c_{0}(\mathcal{S}, \mathcal{T})$, and if $\mathcal{S} \cap \mathcal{T}=\{0\}$ then $c(\mathcal{S}, \mathcal{T})=c_{0}(\mathcal{S}, \mathcal{T})$. Remark 2.1. If $P_{\mathcal{S}}$ and $P_{\mathcal{T}}$ are the orthogonal projections onto $\mathcal{S}$ and $\mathcal{T}$, respectively, then

$$
c_{0}(\mathcal{S}, \mathcal{T})=\left\|P_{\mathcal{S}} P_{\mathcal{T}}\right\| .
$$

Also, $\mathcal{H}=\mathcal{S} \dot{+} \mathcal{T}$ if and only if $\left\|P_{\mathcal{S}^{\perp}} P_{\mathcal{T} \perp}\right\|<1$. See [8] for further details.

\section{Krein spaces}

In what follows we present the standard notation and some basic results on Krein spaces. For a complete exposition on the subject see $[6,12,1]$.

Given a Krein space $(\mathcal{H},[]$,$) with a fundamental decomposition \mathcal{H}=\mathcal{H}_{+} \dot{+}$ $\mathcal{H}_{-}$, the direct (orthogonal) sum of the Hilbert spaces $\left(\mathcal{H}_{+},[],\right)$and $\left(\mathcal{H}_{-},-[],\right)$ is denoted by $(\mathcal{H},\langle\rangle$,$) .$

Observe that the indefinite metric and the inner product of $\mathcal{H}$ are related by means of a fundamental symmetry, i.e. a unitary selfadjoint operator $J \in L(\mathcal{H})$ which satisfies:

$$
[x, y]=\langle J x, y\rangle, \quad x, y \in \mathcal{H} .
$$


If $\mathcal{H}$ and $\mathcal{K}$ are Krein spaces, $L(\mathcal{H}, \mathcal{K})$ stands for the vector space of linear transformations which are bounded with respect to the associated Hilbert spaces $\left(\mathcal{H},\langle,\rangle_{\mathcal{H}}\right)$ and $\left(\mathcal{K},\langle,\rangle_{\mathcal{K}}\right)$. Given $T \in L(\mathcal{H}, \mathcal{K})$, the $J$-adjoint operator of $T$ is defined by $T^{\#}=J_{\mathcal{H}} T^{*} J_{\mathcal{K}}$, where $J_{\mathcal{H}}$ and $J_{\mathcal{K}}$ are the fundamental symmetries associated to $\mathcal{H}$ and $\mathcal{K}$, respectively. An operator $T \in L(\mathcal{H})$ is $J$-selfadjoint if $T=T^{\#}$.

A vector $x \in \mathcal{H}$ is $J$-positive if $[x, x]>0$. A subspace $\mathcal{S}$ of $\mathcal{H}$ is $J$-positive if every $x \in \mathcal{S}, x \neq 0$, is a $J$-positive vector. $J$-nonnegative, $J$-neutral, $J$-negative and $J$-nonpositive vectors and subspaces are defined analogously.

Given a subspace $\mathcal{S}$ of a Krein space $\mathcal{H}$, the $J$-orthogonal complement to $\mathcal{S}$ is defined by

$$
\mathcal{S}^{[\perp]}=\{x \in \mathcal{H}:[x, s]=0, \text { for every } s \in \mathcal{S}\} .
$$

Usually, $\mathcal{S}^{\circ}:=\mathcal{S} \cap \mathcal{S}^{[\perp]}$ (the isotropic part of $\mathcal{S}$ ) is a non-trivial subspace. Then, a subspace $\mathcal{S}$ of $\mathcal{H}$ is $J$-non-degenerated if $\mathcal{S} \cap \mathcal{S}^{[\perp]}=\{0\}$. Otherwise, it is a $J$-degenerated subspace of $\mathcal{H}$.

Definition. A subspace $\mathcal{S}$ of a Krein space $\mathcal{H}$ is a regular subspace if it is the range of a $J$-selfadjoint projection, i.e. if there exists $E \in L(\mathcal{H})$ such that $E=E^{2}=E^{\#}$ and $R(E)=\mathcal{S}$.

Given a regular subspace $\mathcal{S}$, observe that $\mathcal{S}^{[\perp]}$ is the nullspace of the $J$ selfadjoint projection $E$ onto $\mathcal{S}$. Furthermore, if $P$ is the orthogonal projection onto $\mathcal{S}$, the orthogonal projection onto $\mathcal{S}^{[\perp]}$ coincides with $J(I-P) J$. Thus, by $(2.1)$, it follows that

$$
E=P(P+I-J P J)^{-1},
$$

see [3] for another formula for $E$.

Proposition 2.2 ([3]). A closed subspace $\mathcal{S}$ is regular if and only if

$$
\|P J(I-P)\|<1
$$

or equivalently $(I-P) J P J(I-P) \leq(1-\varepsilon) I$ for some $\varepsilon>0$, where $P$ is the orthogonal projection onto $\mathcal{S}$.

The following result seems to be well known, however its proof is included for the sake of completeness.

Lemma 2.3. Let $Q \in L(\mathcal{H})$ be a projection acting on a Krein space $\mathcal{H}$ with fundamental symmetry $J$. Then, the following conditions are equivalent:
1. $Q^{\#} Q=0$;
2. $R(Q)$ is a $J$-neutral subspace;
3. $P J P=0$, where $P$ is the orthogonal projection onto $R(Q)$; 
4. the orthogonal projection $P$ onto $R(Q)$ admits the representation (according to the fundamental decomposition $\left.\mathcal{H}=\mathcal{H}_{+} \oplus \mathcal{H}_{-}\right)$

$$
P=\frac{1}{2}\left(\begin{array}{cc}
V^{*} V & V^{*} \\
V & V V^{*}
\end{array}\right)
$$

where $V \in L\left(\mathcal{H}_{+}, \mathcal{H}_{-}\right)$is a partial isometry.

Proof. The equivalences $1 . \leftrightarrow 2$. $\leftrightarrow 3$. $\leftrightarrow 4$. and the implication 5 . $\rightarrow 1$. are easy to check. On the other hand, if $\mathcal{S}=R(Q)$ is a $J$-neutral subspace of $\mathcal{H}$ then its angular operator $V \in L\left(\mathcal{H}_{+}, \mathcal{H}_{-}\right)$is a partial isometry. Therefore

$$
\begin{aligned}
\mathcal{S} & =\left\{\left(x_{+}, V x_{+}\right) \in \mathcal{H}_{+} \oplus \mathcal{H}_{-}: x_{+} \in P_{+}(\mathcal{S})=N(V)^{\perp}\right\} \\
& =\left\{\left(V^{*} V u, V u\right) \in \mathcal{H}_{+} \oplus \mathcal{H}_{-}: u \in \mathcal{H}_{+}\right\}=R\left(\left[\begin{array}{c}
V V^{*} \\
V
\end{array}\right]\right),
\end{aligned}
$$

see $[12$, Ch. $1, \S 8]$. Then, since $V$ is a partial isometry, the operator

$$
P=\frac{1}{2}\left(\begin{array}{cc}
V^{*} V & V^{*} \\
V & V V^{*}
\end{array}\right)
$$

satisfies $P^{2}=P=P^{*}$, i.e. $P$ is the orthogonal projection onto $\mathcal{S}$.

\section{Decompositions of a $J$-normal projection}

Every normal projection acting on a Hilbert space is selfadjoint. However, the following example shows that there are $J$-normal projections acting on a Krein space (i.e. projections that commute with its $J$-adjoint) which are not $J$-selfadjoint.

Example 1. If $\mathbb{C}^{3}$ is endowed with the indefinite inner product $[x, y]=x_{1} \overline{y_{1}}+$ $x_{2} \overline{y_{2}}-x_{3} \overline{y_{3}}$, where $x=\left(x_{1}, x_{2}, x_{3}\right), y=\left(y_{1}, y_{2}, y_{3}\right) \in \mathbb{C}^{3}$, consider the projection $Q$ whose matrix representation in the canonical basis is given by

$$
Q=\left(\begin{array}{ccc}
1 & 0 & 0 \\
0 & \frac{1}{2} & \frac{1}{2} \\
0 & \frac{1}{2} & \frac{1}{2}
\end{array}\right)
$$

Then, it is easy to see that

$$
Q^{\#}=\left(\begin{array}{rrr}
1 & 0 & 0 \\
0 & \frac{1}{2} & -\frac{1}{2} \\
0 & -\frac{1}{2} & \frac{1}{2}
\end{array}\right) \neq Q \quad \text { and } \quad Q Q^{\#}=\left(\begin{array}{ccc}
1 & 0 & 0 \\
0 & 0 & 0 \\
0 & 0 & 0
\end{array}\right)=Q^{\#} Q
$$

In what follows, the basic properties of $J$-normal projections are developed.

Theorem 3.1. Given a projection $Q \in L(\mathcal{H}), Q$ is $J$-normal if and only if there exist a $J$-selfadjoint projection $E \in L(\mathcal{H})$ and a projection $P \in L(\mathcal{H})$ satisfying $P P^{\#}=P^{\#} P=0$ such that

$$
Q=E+P
$$

The projections $E$ and $P$ are uniquely determined by $Q$. 
Proof. If $Q \in L(\mathcal{H})$ is a $J$-normal projection, then $E=Q Q^{\#}$ is a $J$-selfadjoint projection. Notice that $P:=Q\left(I-Q^{\#}\right)$ is also a projection and, since $I-Q$ is also $J$-normal, it holds that

$$
P P^{\#}=Q\left(I-Q^{\#}\right)(I-Q) Q^{\#}=Q(I-Q)\left(I-Q^{\#}\right) Q^{\#}=0 .
$$

In the same way, $P^{\#} P=0$.

Conversely, suppose that $Q=E+P$ where $E$ is $J$-selfadjoint and $P$ is a projection satisfying $P P^{\#}=P^{\#} P=0$. Since $Q^{2}=Q$, it follows that $E P+P E=0$. Notice that $R(E) \cap R(P)=\{0\}$. In fact, if $x \in R(E) \cap R(P)$ it is easy to see that $0=(E P+P E) x=2 x$. So, $x=0$. Therefore, $E P=P E=0$ (and $E P^{\#}=P^{\#} E=0$ ).

Thus, recalling that $P P^{\#}=P^{\#} P=0$ it follows easily that $Q Q^{\#}=Q^{\#} Q=$ $E$, i.e. $Q$ is $J$-normal. Notice that $P=Q-E=Q\left(I-Q^{\#}\right)$. The uniqueness of this decomposition follows from the last part of the proof.

If $Q \in L(\mathcal{H})$ is a $J$-normal projection, notice that the (uniquely) determined projections in the decomposition of Theorem 3.1 are

$$
E=Q Q^{\#} \quad \text { and } \quad P=Q\left(I-Q^{\#}\right) .
$$

Throughout this paper, $E$ and $P$ will be refered as the regular part and the neutral part of $Q$, respectively.

Corollary 3.2. Let $Q \in L(\mathcal{H})$ be a J-normal projection. Then, $Q$ is $J$ selfadjoint if and only if $R(Q)^{\circ}$ is trivial.

Proof. Observe that $Q$ is $J$-selfadjoint if and only if $Q=Q Q^{\#}$, or equivalently, $P=Q\left(I-Q^{\#}\right)=0$. But $R(P)=R(Q) \cap N\left(Q^{\#}\right)=R(Q)^{\circ}$. So, $P=0$ if and only if $R(Q)^{\circ}=\{0\}$.

Corollary 3.3. Given a projection $Q \in L(\mathcal{H}), Q$ is $J$-normal if and only if

$$
Q=G H,
$$

where $G \in L(\mathcal{H})$ is a $J$-selfadjoint projection and $H \in L(\mathcal{H})$ is a $J$-normal projection with $J$-neutral kernel contained in $R(G)$. Furthermore, this factorization is unique and the projections $G$ and $H$ commute.

Proof. If $Q$ is $J$-normal, then $G=I-(I-Q)(I-Q)^{\#}$ and $H=I-(I-Q) Q^{\#}$ satisfy the desired properties.

Conversely, if $Q=G H$ for a pair of projections $G$ and $H$ satisfying the assumptions, notice that $(I-G)(I-H)=0$, or equivalently, $I+G H=G+H$. Thus,

$$
I-Q=I-G H=(I-G)+(I-H),
$$

$I-G$ is $J$-selfadjoint and $I-H$ satisfies $(I-H)(I-H)^{\#}=(I-H)^{\#}(I-H)=0$. Then, by Theorem 3.1, $Q$ is $J$-normal.

The uniqueness of the factorization and the commutativity of $G$ and $H$ also follow from the above theorem. 
Corollary 3.4. If $Q \in L(\mathcal{H})$ is a $J$-normal projection and $Q=E+P$ is the decomposition given by Theorem 3.1, then there exists a unique J-selfadjoint projection $F \in L(\mathcal{H})$ such that

$$
I-Q=F+P^{\#}
$$

Moreover, $E F=0$.

Proof. Applying Theorem 3.1 to $I-Q$ it follows that its $J$-selfadjoint part is $F=(I-Q)(I-Q)^{\#}$ and

$$
(I-Q)-F=(I-Q)-(I-Q)(I-Q)^{\#}=(I-Q) Q^{\#}=P^{\#} .
$$

Furthermore, $E=Q Q^{\#}=Q^{\#} Q$ and then it is obvious that $E F=0$.

Lemma 3.5. Let $Q \in L(\mathcal{H})$ be a $J$-normal projection and consider the neutral part $P \in L(\mathcal{H})$ of $Q$. Then,

$$
R(P)=R(Q)^{\circ} \quad \text { and } \quad R\left(P^{\#}\right)=N(Q)^{\circ} .
$$

Therefore, $R(Q)^{\circ}$ and $N(Q)^{\circ}$ have the same dimension and codimension.

Proof. Indeed, if $Q$ is $J$-normal then $P=Q\left(I-Q^{\#}\right)=\left(I-Q^{\#}\right) Q$ and

$$
R(P)=R(Q) \cap N\left(Q^{\#}\right)=R(Q) \cap R(Q)^{[\perp]}=R(Q)^{\circ} .
$$

The assertion on $R\left(P^{\#}\right)$ follows analogously. Finally, notice that

$$
\begin{aligned}
\operatorname{dim} R(Q)^{\circ} & =\operatorname{dim} R(P)=\operatorname{dim} N(P)^{\perp}=\operatorname{dim} R\left(P^{*}\right)=\operatorname{dim} R\left(P^{\#}\right) \\
& =\operatorname{dim} N(Q)^{\circ},
\end{aligned}
$$

and $\operatorname{codim} R(Q)^{\circ}=\operatorname{dim} N(P)=\operatorname{dim} R(P)^{\perp}=\operatorname{dim} N\left(P^{*}\right)=\operatorname{dim} N\left(P^{\#}\right)=$ $\operatorname{codim} N(Q)^{\circ}$.

Remark 3.6. Let $Q \in L(\mathcal{H})$ be a $J$-normal projection with decompositions $Q=E+P$ and $I-Q=F+P^{\#}$. From the $J$-normality of $Q$ and the formulas

$$
E=Q Q^{\#}, \quad P=Q\left(I-Q^{\#}\right), \quad F=(I-Q)(I-Q)^{\#} \quad \text { and } \quad P E=P F=0,
$$

the following facts are easily deduced:

1. $R(E)=R(Q) \cap R\left(Q^{\#}\right)$ and $R(F)=N(Q) \cap N\left(Q^{\#}\right)$. Moreover,

$$
R(Q)=R(E)[\dot{+}] R(P) \quad \text { and } \quad N(Q)=R(F)[\dot{+}] R\left(P^{\#}\right) .
$$

2. Also, since $P P^{\#}=P^{\#} P=0$, observe that $P+P^{\#}$ is a $J$-selfadjoint projection with range $R(Q)^{\circ} \dot{+} N(Q)^{\circ}$. Therefore, $R(Q)^{\circ} \dot{+} N(Q)^{\circ}$ is regular. 
3. Finally, by the items above, notice that

$$
\mathcal{H}=R(Q) \dot{+} N(Q)=(R(E)[\dot{+}] R(P)) \dot{+}\left(R(F)[\dot{+}] R\left(P^{\#}\right)\right) .
$$

Then, if $Q$ is $J$-normal, $\mathcal{H}$ can be decomposed as

$$
\mathcal{H}=R(Q) \cap R\left(Q^{\#}\right)[\dot{+}]\left(R(Q)^{\circ} \dot{+} N(Q)^{\circ}\right)[\dot{+}] N(Q) \cap N\left(Q^{\#}\right) .
$$

In fact, (3.5) is equivalent to the $J$-normality of $Q$.

Proposition 3.7. Let $Q \in L(\mathcal{H})$ be a projection. Then, $Q$ is $J$-normal if and only if

$$
\mathcal{H}=R(Q) \cap R\left(Q^{\#}\right) \dot{+} R(Q) \cap N\left(Q^{\#}\right) \dot{+} N(Q) \cap R\left(Q^{\#}\right) \dot{+} N(Q) \cap N\left(Q^{\#}\right) .
$$

Proof. If $Q$ is $J$-normal, the decomposition follows from item 3. in the above remark. Conversely, suppose that (3.6) holds. Given $x \in \mathcal{H}$ there exist (unique) $x_{1} \in R(Q) \cap R\left(Q^{\#}\right), x_{2} \in R(Q) \cap N\left(Q^{\#}\right), x_{3} \in N(Q) \cap R\left(Q^{\#}\right)$ and $x_{4} \in$ $N(Q) \cap N\left(Q^{\#}\right)$ such that $x=x_{1}+x_{2}+x_{3}+x_{4}$. Then,

$$
Q^{\#} Q x=Q^{\#}\left(x_{1}+x_{2}\right)=x_{1}=Q\left(x_{1}+x_{3}\right)=Q Q^{\#} x .
$$

Therefore, $Q^{\#} Q x=Q Q^{\#} x$ for every $x \in \mathcal{H}$, i.e. $Q$ is $J$-normal.

\section{The range of a $J$-normal projection}

The aim of this section is to characterize the ranges of the family of $J$-normal projections acting on a Krein space. The main result in this direction addresses the fact that a (closed) subspace is the range of a $J$-normal projection if and only if it is a pseudo-regular subspace. Thus, the first paragraphs are devoted to recall the definition of pseudo-regularity and to state some well known equivalent conditions. Throughout this section, $\mathcal{H}$ denotes a Krein space with fundamental symmetry $J$.

Definition. A closed subspace $\mathcal{S}$ of $\mathcal{H}$ is called pseudo-regular if the algebraic sum $\mathcal{S}+\mathcal{S}^{[\perp]}$ is closed.

The following proposition compiles several conditions which are equivalent to pseudo-regularity. These facts are well known but they are scattered throughout the literature and different research papers, e.g. see [12, 5, 9, 13].

Proposition 4.1. Let $\mathcal{S}$ be a closed subspace of $\mathcal{H}$ and consider its Gramian operator $G_{\mathcal{S}}=\left.P_{\mathcal{S}} J\right|_{\mathcal{S}}: \mathcal{S} \rightarrow \mathcal{S}$. Then, the following conditions are equivalent:

1. $\mathcal{S}$ is pseudo-regular.

2. $\left(\mathcal{S}^{\circ}\right)^{[\perp]}=\mathcal{S}+\mathcal{S}^{[\perp]}$.

3. There exists a regular subspace $\mathcal{M}$ such that $\mathcal{S}=\mathcal{S}^{\circ}[\dot{+}] \mathcal{M}$. 
4. If $\mathcal{S}=\mathcal{T}+\mathcal{S}^{\circ}$, then $\mathcal{T}$ is regular.

5. There exists a regular subspace $\mathcal{N} \supseteq \mathcal{S}$ such that $\mathcal{S}^{\circ}=\mathcal{N} \cap \mathcal{S}^{[\perp]}$.

6. $\mathcal{S} / \mathcal{S}^{\circ}$ is a Krein space.

7. 0 is an isolated point of $\sigma\left(G_{\mathcal{S}}\right)$.

Proposition 4.2 (T. Ando). Given a (closed) subspace $\mathcal{S}$ of $\mathcal{H}$, consider its isotropic part $\mathcal{S}^{\circ}$. Let $P$ and $P_{0}$ denote the orthogonal projections onto $\mathcal{S}$ and $\mathcal{S}^{\circ}$, respectively. Then, $\mathcal{S}$ is pseudo-regular if and only if

$$
\left\|\left(P-P_{0}\right) J(I-P)\right\|<1 .
$$

Proof. Observe that $J(I-P) J$ is the orthogonal projection onto $\mathcal{S}^{[\perp]}$. By definition, $\mathcal{S}$ is pseudo-regular if

$$
\mathcal{S}+\mathcal{S}^{[\perp]} \text { is closed. }
$$

But $\mathcal{S}+\mathcal{S}^{[\perp]}$ is closed if and only if $c\left(\mathcal{S}, \mathcal{S}^{[\perp]}\right)<1$. Also, notice that $c\left(\mathcal{S}, \mathcal{S}^{[\perp]}\right)=$ $c_{0}\left(\mathcal{S} \ominus \mathcal{S}^{\circ}, \mathcal{S}^{[\perp]}\right)=\left\|\left(P-P_{0}\right) J(I-P) J\right\|$ (see the Preliminaries). Hence, $\mathcal{S}$ is pseudo-regular if and only if

$$
\left\|\left(P-P_{0}\right) J(I-P)\right\|<1 .
$$

Theorem 4.3. Let $\mathcal{S}$ be a closed subspace of $\mathcal{H}$. Then, $\mathcal{S}$ is the range of a $J$-normal projection if and only if $\mathcal{S}$ is a pseudo-regular subspace of $\mathcal{H}$.

Proof. If $\mathcal{S}$ is the range of a $J$-normal projection $Q$ then, by Remark 3.6, $\mathcal{S}=$ $R(E)[+] \mathcal{S}^{\circ}$ where $E=Q Q^{\#}$. Furthermore, $R(E)$ is regular because $E$ is a $J$-selfadjoint projection. Thus, $\mathcal{S}$ is a pseudo-regular subspace.

Conversely, suppose that $\mathcal{S}$ is a pseudo-regular subspace and let $P$ be the orthogonal projection onto the isotropic subspace $\mathcal{S}^{\circ}$. Since $R(P)$ is $J$-neutral, it follows by Lemma 2.3 that $P J P=0$. Then, $P P^{\#}=P^{\#} P=0$.

Consider the subspace $\mathcal{T}=\mathcal{S} \ominus \mathcal{S}^{\circ}$. Since $\mathcal{S}=\mathcal{T}[\dot{+}] \mathcal{S}^{\circ}$, Proposition 4.1 assures that $\mathcal{T}$ is a regular subspace of $\mathcal{H}$. Thus, there is a (unique) $J$-selfadjoint projection $E$ with $R(E)=\mathcal{T}$.

Furthermore, $P E=E P=0$ because $\mathcal{T} \subset\left(\mathcal{S}^{\circ}\right)^{\perp}$ and $\mathcal{S}^{\circ} \subset \mathcal{S}^{[\perp]} \subset \mathcal{T}^{[\perp]}$. Then $Q=E+P$ is also a projection with

$$
R(Q)=R(E)+R(P)=\mathcal{T} \dot{+} \mathcal{S}^{\circ}=\mathcal{S} .
$$

Finally, the $J$-normality of $Q$ follows from Theorem 3.1.

Recall that if $\kappa=\min \left\{\operatorname{dim} \mathcal{H}_{+}, \operatorname{dim} \mathcal{H}_{-}\right\}<\infty$, the Krein space with fundamental decomposition $\mathcal{H}=\mathcal{H}_{+} \dot{+} \mathcal{H}_{-}$is called a Pontryagin space and is denoted by $\Pi_{\kappa}$. In a Pontryagin space $\Pi_{\kappa}$, a closed subspace $\mathcal{S}$ is regular if and only if it is $J$-non-degenerated (see e.g. [12]). Thus, every $J$-non-degenerated subspace of $\Pi_{\kappa}$ admits a (unique) $J$-selfadjoint projection onto it. Furthermore, 
Corollary 4.4. If $\Pi_{\kappa}$ is a Pontryagin space, then every closed subspace $\mathcal{S}$ of $\Pi_{\kappa}$ admits a $J$-normal projection onto it.

Proof. Since $\mathcal{S}^{\circ}$ is a closed subspace of $\mathcal{S}, \mathcal{S}$ can be written as

$$
\mathcal{S}=\mathcal{S}^{\circ} \oplus\left(\mathcal{S} \ominus \mathcal{S}^{\circ}\right) .
$$

Furthermore, $\mathcal{T}:=\mathcal{S} \ominus \mathcal{S}^{\circ}$ is $J$-orthogonal to $\mathcal{S}^{\circ}$. Hence, $\mathcal{S}=\mathcal{S}^{\circ}[\dot{+}] \mathcal{T}$. It is easy to see that $\mathcal{T}$ is a $J$-non-degenerated subspace of $\mathcal{H}$ and therefore, $\mathcal{T}$ is regular because $\Pi_{\kappa}$ is a Pontryagin space. Thus, $\mathcal{S}$ is the direct sum of its isotropic part and a regular subspace and, by Theorem $4.3, \mathcal{S}$ is the range of a $J$-normal projection.

The last paragraphs of this section are devoted to discussing the non-uniqueness of $J$-normal projections associated to a pseudo-regular subspace. First of all, observe the following example.

Example 2. As in Example 1, consider the Minkowski space $\left(\mathbb{C}^{3},[],\right)$. Fix $\mathcal{S}$ by $\mathcal{S}=\operatorname{span}\{(1,0,0),(0,1,1)\}$. Given a vector $v=(x, y, z) \in \mathbb{C}^{3} \backslash \mathcal{S}$, let $Q_{v}$ be the projection onto $\mathcal{S}$ along the subspace spanned by $v$. According to the canonical basis of $\mathbb{C}^{3}$, its matrix representation is

$$
Q_{v}=\frac{1}{z-y}\left(\begin{array}{ccc}
z-y & x & -x \\
0 & z & -y \\
0 & z & -y
\end{array}\right) .
$$

A few calculations show that

$$
Q_{v}^{\#}=\frac{1}{\overline{z-y}}\left(\begin{array}{ccc}
\overline{z-y} & 0 & 0 \\
\bar{x} & \bar{z} & -\bar{z} \\
\bar{x} & \bar{y} & -\bar{y}
\end{array}\right) .
$$

Then, it is easy to see that

$$
\begin{aligned}
Q_{v}^{\#} Q_{v} & =\frac{1}{|z-y|^{2}}\left(\begin{array}{ccc}
|z-y|^{2} & \overline{x(z-y)} & -x \overline{(z-y)} \\
\bar{x}(z-y) & |x|^{2} & -\left.x\right|^{2} \\
\bar{x}(z-y) & |x|^{2} & -|x|^{2}
\end{array}\right) \text { and } \\
Q_{v} Q_{v}^{\#} & =\frac{1}{|z-y|^{2}}\left(\begin{array}{ccc}
|z-y|^{2} & x \bar{x}(\bar{y}-y) & -x \overline{(z-y)} \\
\bar{x}(z-y) & |z|^{2}-|y|^{2} & -|z|^{2}+|y|^{2} \\
\bar{x}(z-y) & |z|^{2}-|y|^{2} & -|z|^{2}+|y|^{2}
\end{array}\right) .
\end{aligned}
$$

Therefore, $Q_{v}$ is a $J$-normal projection onto $\mathcal{S}$ if and only if $|z|^{2}=|x|^{2}+|y|^{2}$.

The above example also shows that, for a fixed projection $Q \in L(\mathcal{H})$, the idempotency of the $J$-selfadjoint operators $Q Q^{\#}$ and $Q^{\#} Q$ is not a sufficient condition for the $J$-normality of $Q$. In fact, notice that $Q_{v}^{\#} Q_{v}$ and $Q_{v} Q_{v}^{\#}$ are projections for every $v \in \mathbb{C}^{3} \backslash \mathcal{S}$, even if $|z|^{2} \neq|x|^{2}+|y|^{2}$.

Although there is not a unique $J$-normal projection onto a fixed arbitrary pseudo-regular subspace $\mathcal{S}$, it is possible to present a particular $J$-normal projection onto $\mathcal{S}$ in terms of the orthogonal projections onto $\mathcal{S}$ and $\mathcal{S}^{\circ}$. Observe that this particular $J$-normal projection onto $\mathcal{S}$ is the one discussed in Theorem 4.3 . 
Corollary 4.5. Given a (closed) pseudo-regular subspace $\mathcal{S}$ of $\mathcal{H}$, let $P$ and $P_{0}$ denote the orthogonal projections onto $\mathcal{S}$ and $\mathcal{S}^{\circ}$, respectively. Then,

$$
Q=\left(P-P_{0}\right)\left(P-P_{0}+I-J\left(P-P_{0}\right) J\right)^{-1}+P_{0},
$$

is a J-normal projection onto $\mathcal{S}$.

Proof. Since $\mathcal{S} \ominus \mathcal{S}^{\circ}$ is a regular subspace of $\mathcal{H}$, the $J$-selfadjoint projection $E$ onto $\mathcal{S} \ominus \mathcal{S}^{\circ}$ can be written as

$$
E=\left(P-P_{0}\right)\left(P-P_{0}+I-J\left(P-P_{0}\right) J\right)^{-1},
$$

see (2.2). Furthermore, by Theorem 3.1, $Q=E+P_{0}=\left(P-P_{0}\right)\left(P-P_{0}+I-\right.$ $\left.J\left(P-P_{0}\right) J\right)^{-1}+P_{0}$ is a $J$-normal projection onto $\mathcal{S}$.

\section{$5 J$-normal projections with $J$-neutral range}

From now on, every subspace considered is assumed to be closed.

As it was shown in the previous section, a pseudo-regular subspace may admit infinitely many $J$-normal projections onto it. In order to provide a parametrization of the set of $J$-normal projections onto a prescribed pseudoregular subspace, consider the simplest case first, i.e. a $J$-neutral subspace. This section is devoted to studying $J$-normal projections onto $J$-neutral subspaces, i.e. those projections $P \in L(\mathcal{H})$ satisfying $P P^{\#}=P^{\#} P=0$.

It is obvious that every $J$-neutral subspace $\mathcal{N}$ of a Krein space $\mathcal{H}$ is a pseudoregular one, since $\mathcal{N}=\mathcal{N}^{\circ}$. In particular,

Lemma 5.1. If $\mathcal{N}$ is a $J$-neutral subspace then the orthogonal projection $P:=$ $P_{\mathcal{N}} \in L(\mathcal{H})$ is $J$-normal. Furthermore, $P P^{\#}=P^{\#} P=0$.

Proof. By Lemma 2.3, the assumption on $\mathcal{N}$ is equivalent to $P J P=0$. Thus,

$$
P P^{\#}=P J P \cdot J=0 \quad \text { and } \quad P^{\#} P=J \cdot P J P=0 .
$$

Proposition 5.2. Let $\mathcal{N}_{1}$ and $\mathcal{N}_{2}$ be (closed) J-neutral subspaces of $\mathcal{H}$ such that $\mathcal{N}_{1} \cap \mathcal{N}_{2}=\{0\}$. Then, the following conditions are equivalent:

1. there exists a $J$-normal projection $P \in L(\mathcal{H})$ such that $R(P)=\mathcal{N}_{1}$ and $R\left(P^{\#}\right)=\mathcal{N}_{2}$;

2. $\mathcal{N}_{1}+\mathcal{N}_{2}$ is regular;

3. $\mathcal{N}_{1}+\mathcal{N}_{2}^{[\perp]}=\mathcal{H}$.

Proof. 1. $\Rightarrow$ 2. follows from item 2. of Remark 3.6.

2. $\Rightarrow$ 3.: Suppose that $\mathcal{M}=\mathcal{N}_{1}+\mathcal{N}_{2}$ is regular. Then, $\mathcal{M}^{[\perp]}=\mathcal{N}_{1}^{[\perp]} \cap \mathcal{N}_{2}^{[\perp]}$ is also regular and

$$
\mathcal{H}=\mathcal{M}+\mathcal{M}^{[\perp]}=\mathcal{N}_{1}+\left(\mathcal{N}_{2}+\mathcal{N}_{1}^{[\perp]} \cap \mathcal{N}_{2}^{[\perp]}\right) \subseteq \mathcal{N}_{1}+\mathcal{N}_{2}^{[\perp]},
$$


because $\mathcal{N}_{2}$ is $J$-neutral. Analogously, $\mathcal{H}=\mathcal{N}_{1}^{[\perp]}+\mathcal{N}_{2}$ and $\mathcal{N}_{1} \cap \mathcal{N}_{2}^{[\perp]}=\left(\mathcal{N}_{1}^{[\perp]}+\right.$ $\left.\mathcal{N}_{2}\right)^{[\perp]}=\{0\}$. Thus, $\mathcal{H}=\mathcal{N}_{1}+\mathcal{N}_{2}^{[\perp]}$.

3. $\Rightarrow$ 1.: If $\mathcal{N}_{1} \dot{+} \mathcal{N}_{2}^{[\perp]}=\mathcal{H}$, consider the projection $P:=P_{\mathcal{N}_{1} / / \mathcal{N}_{2}^{[\perp]}}$. Then, $P^{\#}=P_{\mathcal{N}_{2} / / \mathcal{N}_{1}^{[\perp]}}$ and it is easy to see that $P P^{\#}=P^{\#} P=0$. Therefore, $P$ is a $J$-normal projection with $R(P)=\mathcal{N}_{1}$ and $R\left(P^{\#}\right)=\mathcal{N}_{2}$.

As a consequence of the above proposition, if $P$ is a $J$-normal projection onto a $J$-neutral subspace, the subspaces $R(P)$ and $R\left(P^{\#}\right)$ are skewly linked (see [12, Def. 1.29]). Moreover, in a Pontryagin space $\Pi_{\kappa}$, a pair of $J$-neutral subspaces $\mathcal{N}_{1}, \mathcal{N}_{2}$ of $\Pi_{\kappa}$ is skewly linked if and only if there exists a $J$-normal projection $P \in L(\mathcal{H})$ such that $R(P)=\mathcal{N}_{1}$ and $R\left(P^{\#}\right)=\mathcal{N}_{2}$.

Remark 5.3. If $\mathcal{N}$ is a $J$-neutral subspace then $\mathcal{N}+J(\mathcal{N})$ is regular. In fact, by Lemma 5.1, the orthogonal projection $P$ onto $\mathcal{N}$ is a $J$-normal projection and $R\left(P^{\#}\right)=J(\mathcal{N})$. So, by the above proposition, $\mathcal{N}+J(\mathcal{N})$ is regular.

Proposition 5.4. Let $Q \in L(\mathcal{H})$ be a projection such that $R(Q)^{\circ}+N(Q)^{\circ}$ is regular. Then, there exist projections $E, P \in L(\mathcal{H})$ such that $P P^{\#}=P^{\#} P=0$ and

$$
Q=E+P .
$$

Proof. By Proposition 5.2, $\mathcal{H}$ can be decomposed as $\mathcal{H}=R(Q)^{\circ}+\left(N(Q)^{\circ}\right)^{[\perp]}$

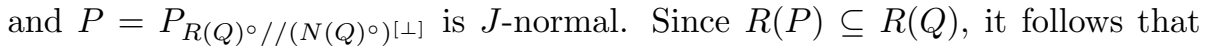
$Q P=P$. Also, $P Q$ is a projection and $R(P Q)=R(P)$. Furthermore,

$$
\begin{aligned}
N(P Q) & =N(Q)+R(Q) \cap N(P)=N(Q)+R(Q) \cap\left(N(Q)^{\circ}\right)^{[\perp]} \\
& \subseteq\left(N(Q)^{\circ}\right)^{[\perp]}=N(P) .
\end{aligned}
$$

Thus, $P Q=P$ and $E:=Q-P$ is a projection because of

$$
E^{2}=Q-Q P-P Q+P=Q-P-P+P=Q-P=E .
$$

Notice that $P E=E P=0$ and therefore $Q=E+P$.

Following the notation of the above proof, observe that $E=Q-P=$ $Q(I-P)=(I-P) Q$. Hence, $R(E)=R(Q) \cap N(P)=R(Q) \cap\left(N(Q)^{\circ}\right)^{[\perp]}$ and $N(E)=R(P)+N(Q)=R(Q)^{\circ}+N(Q)$. Therefore,

$$
E=P_{R(Q) \cap\left(N(Q)^{\circ}\right)^{[\perp]} / / R(Q)^{\circ}+N(Q)} .
$$

Thus, the following is a sufficient condition to guarantee that the decomposition of the above proposition is the same as in Theorem 3.1.

Corollary 5.5. Let $Q \in L(\mathcal{H})$ be a projection such that $R(Q)^{\circ}+N(Q)^{\circ}$ is regular. Then, the following conditions are equivalent:

1. $Q$ is J-normal;

2. $R(Q) \cap\left(N(Q)^{\circ}\right)^{[\perp]} \subseteq R(Q) \cap R\left(Q^{\#}\right)$; 
3. $N(Q) \cap\left(R(Q)^{\circ}\right)^{[\perp]} \subseteq N(Q) \cap N\left(Q^{\#}\right)$.

Proof. If $Q$ is $J$-normal, then $N(Q)$ is a pseudo-regular subspace. So,

$$
\left(N(Q)^{\circ}\right)^{[\perp]}=N(Q)+N(Q)^{[\perp]}=N(Q)+R\left(Q^{\#}\right) .
$$

Then, if $x \in R(Q) \cap\left(N(Q)^{\circ}\right)^{[\perp]}$, there exist $u \in N(Q)$ and $v \in \mathcal{H}$ such that $x=u+Q^{\#} v$. Hence,

$$
x=Q x=Q\left(u+Q^{\#} v\right)=Q Q^{\#} v,
$$

i.e. $x \in R(Q) \cap R\left(Q^{\#}\right)$. Thus, $R(Q) \cap\left(N(Q)^{\circ}\right)^{[\perp]} \subseteq R(Q) \cap R\left(Q^{\#}\right)$.

Conversely, suppose that $R(Q) \cap\left(N(Q)^{\circ}\right)^{[\perp]} \subseteq R(Q) \cap R\left(Q^{\#}\right)$. Then, consider the decomposition $Q=E+P$ given by Proposition 5.4, where $E, P \in L(\mathcal{H})$ are projections and $P P^{\#}=P^{\#} P=0$. Observe that

$$
R(E)=R(Q) \cap\left(N(Q)^{\circ}\right)^{[\perp]}=R(Q) \cap R\left(Q^{\#}\right),
$$

because $N(Q)^{\circ} \subseteq N(Q)=R\left(Q^{\#}\right)^{[\perp]}$. Also,

$$
R\left(E^{\#}\right)=N(E)^{[\perp]}=N(Q)^{[\perp]} \cap\left(R(Q)^{\circ}\right)^{[\perp]} \supseteq R\left(Q^{\#}\right) \cap R(Q)=R(E) .
$$

Thus, $E^{\#} E=E$ and, by Theorem 3.1, $Q$ is $J$-normal.

Finally, notice that the equivalence 1 . $\leftrightarrow 3$. follows considering $I-Q$ instead of $Q$.

The following result shows that, for a fixed $J$-neutral subspace, there are infinitely many $J$-normal projections onto it. Furthermore, the nullspaces of these projections can be arbitrarily close.

Proposition 5.6 (T. Ando). Suppose that a (non-trivial) projection $P \in L(\mathcal{H})$ satisfies $P P^{\#}=P^{\#} P=0$. Then, there exists a one-parameter family of (different) $J$-normal projections $P_{\varepsilon} \in L(\mathcal{H})$ onto $R(P)$ (for $0<\varepsilon<\varepsilon_{0}$ ) such that

$$
\left\|P_{\varepsilon}-P\right\| \rightarrow 0 \quad \text { as } \varepsilon \rightarrow 0 .
$$

Proof. Let $P_{R}\left(\right.$ resp. $\left.P_{N}\right)$ be the orthogonal projection onto $R(P)$ (resp. $N(P)$ ). Then, the ranges of these projections are $J$-neutral subspaces and, by Lemma 2.3 , there is a partial isometry $V \in L\left(\mathcal{H}_{+}, \mathcal{H}_{-}\right)$such that

$$
I-P_{N}=\frac{1}{2}\left(\begin{array}{cc}
V^{*} V & V^{*} \\
V & V V^{*}
\end{array}\right) .
$$

Since $e^{i \varepsilon} V$ is also a partial isometry (for every $\varepsilon>0$ ), there is an orthogonal projection $Q_{\varepsilon}$ such that

$$
I-Q_{\varepsilon}=\frac{1}{2}\left(\begin{array}{cc}
V^{*} V & e^{-i \varepsilon} V^{*} \\
e^{i \varepsilon} V & V V^{*}
\end{array}\right),
$$

so that $\left(I-Q_{\varepsilon}\right) J\left(I-Q_{\varepsilon}\right)=0$. It is clear that $\left\|P_{N}-Q_{\varepsilon}\right\| \rightarrow 0$ as $\varepsilon \rightarrow 0$. 
that

Since $\left\|P_{R} P_{N}\right\|<1$ and $\left\|\left(I-P_{R}\right)\left(I-P_{N}\right)\right\|<1$, there exists $\varepsilon_{0}>0$ such

$$
\left\|P_{R} Q_{\varepsilon}\right\|<1 \text { and }\left\|\left(I-P_{R}\right)\left(I-Q_{\varepsilon}\right)\right\|<1 \quad \text { for } 0<\varepsilon \leq \varepsilon_{0} .
$$

Hence, there is a projection $P_{\varepsilon} \in L(\mathcal{H})$ with $R\left(P_{\varepsilon}\right)=R(P)$ and $N\left(P_{\varepsilon}\right)=R\left(Q_{\varepsilon}\right)$, see Remark 2.1. Then, by Lemma 2.3, $P_{\varepsilon} P_{\varepsilon}^{\#}=P_{\varepsilon}^{\#} P_{\varepsilon}=0$. Finally, $P_{\varepsilon}$ can be represented as:

$$
P_{\varepsilon}=P_{R}\left(P_{R}+Q_{\varepsilon}\right)^{-1},
$$

see (2.1). So, $P_{\varepsilon} \neq P$ for every $0<\varepsilon \leq \varepsilon_{0}$, and $\left\|P_{\varepsilon}-P\right\| \rightarrow 0$ as $\varepsilon \rightarrow 0$.

Corollary 5.7. Suppose that a (non-trivial) projection $P \in L(\mathcal{H})$, satisfies $P P^{\#}=P^{\#} P=0$. Then, there exists a one-parameter family of (different) $J$-normal projections $P_{\varepsilon} \in L(\mathcal{H})$ onto $R(P)$ (for $0<\varepsilon<\varepsilon_{0}$ ) such that

$$
c\left(N(P), N\left(P_{\varepsilon}\right)\right) \longrightarrow 1 \quad \text { as } \varepsilon \rightarrow 0 .
$$

Proof. Consider the projections $P_{\varepsilon}$ obtained in Proposition 5.6. Following the notations in the proof above, $N(P)=R\left(P_{N}\right)$ and $N\left(P_{\varepsilon}\right)=R\left(Q_{\varepsilon}\right)$. Then,

$$
c\left(N(P), N\left(P_{\varepsilon}\right)\right)=c\left(R\left(P_{N}\right), R\left(Q_{\varepsilon}\right)\right)=c\left(R\left(I-P_{N}\right), R\left(I-Q_{\varepsilon}\right)\right),
$$

because $P_{N}$ and $Q_{\varepsilon}$ are orthogonal projections. By Remark 2.1,

$$
\begin{aligned}
& c\left(R\left(I-P_{N}\right), R\left(I-Q_{\varepsilon}\right)\right)^{2}= \\
= & \left\|\left(I-Q_{\varepsilon}\right)\left(I-P_{N}\right)\right\|^{2}=\left\|\left(I-Q_{\varepsilon}\right)\left(I-P_{N}\right)\left(I-Q_{\varepsilon}\right)\right\|= \\
= & \frac{\left|\left(1+e^{i \varepsilon}\right)\left(1+e^{-i \varepsilon}\right)\right|}{4}\left\|\frac{1}{2}\left(\begin{array}{cc}
V^{*} V & \frac{1+e^{-i \varepsilon}}{1+e^{i \varepsilon}} V^{*} \\
\frac{1+e^{i \varepsilon}}{1+e^{-i \varepsilon}} V & V V^{*}
\end{array}\right)\right\|= \\
= & \frac{\left|\left(1+e^{i \varepsilon}\right)\left(1+e^{-i \varepsilon}\right)\right|}{4}=\frac{1+\cos (\varepsilon)}{2}=\cos ^{2}\left(\frac{\varepsilon}{2}\right) .
\end{aligned}
$$

Therefore, $c\left(N(P), N\left(P_{\varepsilon}\right)\right)=\cos \left(\frac{\varepsilon}{2}\right) \longrightarrow 1$ as $\varepsilon \rightarrow 0$.

\section{$J$-normal projections with prescribed $J$-neutral range}

Let $\mathcal{N}$ be a $J$-neutral subspace of a Krein space $\mathcal{H}$ with fundamental symmetry $J$. Along these paragraphs, a parametrization for the set of $J$-normal projections onto $\mathcal{N}$ is presented. These results are generalized to an arbitrary pseudo-regular subspace in Section 6 .

According to the orthogonal decomposition $\mathcal{H}=\mathcal{N} \oplus \mathcal{N}^{\perp}$, the symmetry $J$ can be written as a block-operator-matrix

$$
J=\left(\begin{array}{cc}
0 & a \\
a^{*} & b
\end{array}\right) \begin{aligned}
& \mathcal{N} \\
& \mathcal{N}^{\perp}
\end{aligned}
$$

where $a \in L\left(\mathcal{N}^{\perp}, \mathcal{N}\right)$ and $b=b^{*} \in L\left(\mathcal{N}^{\perp}\right)$ satisfy

$$
a a^{*}=I_{\mathcal{N}}, \quad a b=0 \quad \text { and } \quad a^{*} a+b^{2}=I_{\mathcal{N} \perp} .
$$


Since $a \in L\left(\mathcal{N}^{\perp}, \mathcal{N}\right)$ is a coisometry, it follows that $a^{*} \in L\left(\mathcal{N}, \mathcal{N}^{\perp}\right)$ is a partial isometry with final space:

$$
R\left(a^{*} a\right)=R\left(a^{*}\right)=J(\mathcal{N}) .
$$

Thus, $a^{*} a \in L\left(\mathcal{N}^{\perp}\right)$ is the orthogonal projection onto $J(\mathcal{N})$.

On the other hand, if $P$ is a projection with range $\mathcal{N}$ then $P$ can be written as a block-operator-matrix

$$
P=\left(\begin{array}{ll}
I & x \\
0 & 0
\end{array}\right)
$$

with $x \in L\left(\mathcal{N}^{\perp}, \mathcal{N}\right)$. Furthermore, $P$ satisfies $P P^{\#}=0$ if and only if

$$
0=\left(\begin{array}{ll}
I & x \\
0 & 0
\end{array}\right)\left(\begin{array}{cc}
0 & a \\
a^{*} & b
\end{array}\right)\left(\begin{array}{cc}
I & 0 \\
x^{*} & 0
\end{array}\right)=\left(\begin{array}{cc}
a x^{*}+x a^{*}+x b x^{*} & 0 \\
0 & 0
\end{array}\right)
$$

or equivalently, $x \in L\left(\mathcal{N}^{\perp}, \mathcal{N}\right)$ is a solution of the equation

$$
a x^{*}+x a^{*}+x b x^{*}=0 .
$$

Thus, in order to describe the set of $J$-normal projections onto the $J$-neutral subspace $\mathcal{N}$, the above equation has to be solved. The following result provides a parametrization for the set of solutions of (5.3).

Lemma 5.8. Let $\mathcal{N}$ be a J-neutral subspace of $\mathcal{H}$. Then, $x \in L\left(\mathcal{N}^{\perp}, \mathcal{N}\right)$ is a solution of (5.3) if and only if there exist operators $A \in L(\mathcal{N})$ and $B \in$ $L\left(\mathcal{N}^{\perp}, \mathcal{N}\right)$ such that $A$ is antihermitian, $J(\mathcal{N}) \subseteq N(B)$ and

$$
x=\left(A-\frac{1}{2} B b B^{*}\right) a+B .
$$

Proof. Recall that the operators $a$ and $b$ considered in (5.3) satisfy the conditions in (5.2). First, suppose that $x \in L\left(\mathcal{N}^{\perp}, \mathcal{N}\right)$ is a solution of (5.3). Since $a^{*} a+$ $b^{2}=I_{\mathcal{N}^{\perp}}, x$ can be written as $x=x_{1}+x_{2}$, where $x_{1}=x a^{*} a$ and $x_{2}=x b^{2}$.

Observe that $x_{2} a^{*}=x_{1} b=0$. Thus, $0=a x^{*}+x a^{*}+x b x^{*}=a x_{1}^{*}+x_{1} a^{*}+$ $x_{2} b x_{2}^{*}$. In other words,

$$
2 \operatorname{Re}\left(x_{1} a^{*}\right)=a x_{1}^{*}+x_{1} a^{*}=-x_{2} b x_{2}^{*} .
$$

So, the antihermitian operator $A=i \operatorname{Im}\left(x_{1} a^{*}\right) \in L(\mathcal{N})$ satisfies

$$
x_{1}=x_{1} a^{*} a=\left(A-\frac{1}{2} x_{2} b x_{2}^{*}\right) a .
$$

Then, considering $B=x_{2}=x\left(I_{\mathcal{N}^{\perp}}-a^{*} a\right) \in L\left(\mathcal{N}^{\perp}, \mathcal{N}\right)$ it follows that $J(\mathcal{N}) \subseteq$ $N(B)$ and

$$
x=\left(A-\frac{1}{2} B b B^{*}\right) a+B .
$$

Conversely, given an antihermitian operator $A \in L(\mathcal{N})$ and $B \in L\left(\mathcal{N}^{\perp}, \mathcal{N}\right)$ such that $J(\mathcal{N}) \subseteq N(B)$, consider

$$
x:=\left(A-\frac{1}{2} B b B^{*}\right) a+B .
$$


Then, it is easy to see that $x a^{*}=A-\frac{1}{2} B b B^{*}$ and $x b x^{*}=B b B^{*}$. Therefore,

$$
x a^{*}+a x^{*}+x b x^{*}=\left(A-\frac{1}{2} B b B^{*}\right)+\left(-A-\frac{1}{2} B b B^{*}\right)+B b B^{*}=0,
$$

i.e. $x \in L\left(\mathcal{N}^{\perp}, \mathcal{N}\right)$ is a solution of (5.3).

Proposition 5.9. Let $\mathcal{N}$ be a J-neutral subspace of $\mathcal{H}$. Then, $P \in L(\mathcal{H})$ is a $J$-normal projection onto $\mathcal{N}$ if and only if there exist $A=-A^{*} \in L(\mathcal{N})$ and $B \in L\left(\mathcal{N}^{\perp}, \mathcal{N}\right)$ with $J(\mathcal{N}) \subseteq N(B)$ such that

$$
P=\left(\begin{array}{cc}
I & \left(A-\frac{1}{2} B b B^{*}\right) a+B \\
0 & 0
\end{array}\right),
$$

according to the orthogonal decomposition $\mathcal{H}=\mathcal{N} \oplus \mathcal{N}^{\perp}$.

\section{A parametrization for the set of $J$-normal pro- jections}

Let $\mathcal{S}$ be a pseudo-regular subspace of a Krein space $\mathcal{H}$ with fundamental symmetry $J$, and denote

$$
\mathcal{Q}_{\mathcal{S}}=\left\{Q \in L(\mathcal{H}): Q^{2}=Q, Q Q^{\#}=Q^{\#} Q \text { and } R(Q)=\mathcal{S}\right\} .
$$

The aim of this section is to present an explicit parametrization of $\mathcal{Q}_{\mathcal{S}}$. First, notice that there are as many projections in $\mathcal{Q}_{\mathcal{S}}$ as in $\mathcal{Q}_{\mathcal{S}^{\circ}}$.

Lemma 6.1. Suppose that $\mathcal{S}$ is a pseudo-regular subspace of $\mathcal{H}$. If $P$ is a $J$ normal projection onto $\mathcal{S}^{\circ}$ then there is a unique $J$-normal projection $Q$ onto $\mathcal{S}$ such that $P$ is the neutral part of $Q$, i.e. $P=Q(I-Q)^{\#}$.

Proof. Suppose that $\mathcal{S}$ is a pseudo-regular subspace of $\mathcal{H}$ and consider $\mathcal{T}=\mathcal{S} \cap$ $N(P)$. Since $P$ is a projection onto $\mathcal{S}^{\circ} \subseteq \mathcal{S}$, given $s \in \mathcal{S},(I-P) s \in \mathcal{S}+\mathcal{S}^{\circ}=\mathcal{S}$. So that, $(I-P) s \in \mathcal{S} \cap N(P)$. Therefore,

$$
\mathcal{S}=\mathcal{S}^{\circ}+\mathcal{T}
$$

Then, by Proposition 4.1, $\mathcal{T}$ is a regular subspace of $\mathcal{H}$. Let $E$ be the $J$ selfadjoint projection onto $\mathcal{T}$.

Notice that $E P=0$ because $\mathcal{S}^{\circ} \subseteq \mathcal{S}^{[\perp]} \subseteq \mathcal{T}^{[\perp]}$. On the other hand, $R(E)=\mathcal{T} \subseteq N(P)$. So, $P E=0$ and, since $E$ is $J$-selfadjoint, the following commutativity relations have been established:

$$
E P=P E=0 \text { and } E P^{\#}=P^{\#} E=0 .
$$

Now, define $Q=E+P$. Then, by Theorem 3.1, $Q$ is a $J$-normal projection and $P=Q-E=Q-Q Q^{\#}=Q\left(I-Q^{\#}\right)$.

Finally, suppose that there is another $J$-normal projection $Q^{\prime} \in L(\mathcal{H})$ onto $\mathcal{S}$ such that $P=Q^{\prime}\left(I-Q^{\prime}\right)^{\#}$. Then, $E^{\prime}=Q^{\prime}-P=Q^{\prime}\left(Q^{\prime}\right)^{\#}$ is a $J$-selfadjoint 
projection onto a subspace of $\mathcal{S}$. Notice that $R\left(E^{\prime}\right) \subseteq N(P)$ because $P E^{\prime}=0$. Hence, $R\left(E^{\prime}\right) \subseteq \mathcal{T}$. But,

$$
R\left(E^{\prime}\right) \dot{+} \mathcal{S}^{\circ}=\mathcal{S}=\mathcal{T} \dot{+} \mathcal{S}^{\circ} .
$$

Thus, $R\left(E^{\prime}\right)=\mathcal{T}$ and, by the uniqueness of the $J$-selfadjoint projection onto a regular subspace, $E^{\prime}=E$.

Theorem 6.2. Given a pseudo-regular subspace $\mathcal{S}$ of $\mathcal{H}$ with isotropic part $\mathcal{S}^{\circ}$, there is a (continuous) bijection between $\mathcal{Q}_{\mathcal{S}}$ and $\mathcal{Q}_{\mathcal{S}}$.

Proof. For a fixed pseudo-regular subspace $\mathcal{S}$ of $\mathcal{H}$, let $\Phi: \mathcal{Q}_{\mathcal{S}} \rightarrow \mathcal{Q}_{\mathcal{S}}$ be defined by

$$
\Phi(Q)=Q\left(I-Q^{\#}\right) .
$$

It follows by the above lemma that $\Phi$ is bijective, because for every $P \in \mathcal{Q}_{\mathcal{S}^{\circ}}$ there exists a unique $Q \in \mathcal{Q}_{\mathcal{S}}$ such that $\Phi(Q)=P$.

Corollary 6.3. Let $\mathcal{S}$ be a pseudo-regular subspace of a Krein space $\mathcal{H}$ with fundamental symmetry $J$. Then, there is a unique $J$-normal projection $Q$ onto $\mathcal{S}$ if and only if $\mathcal{S}^{\circ}=\{0\}$. Moreover, in this case $Q$ is $J$-selfadjoint.

Proof. If $\mathcal{S}^{\circ}=\{0\}$ then $\mathcal{S}$ is a regular subspace and there exists a (unique) $J$-selfadjoint projection onto $\mathcal{S}$. Moreover, if $Q$ is a $J$-normal projection onto $\mathcal{S}$ then, by Theorem 3.1, $Q=E+P$ where $E$ is $J$-selfadjoint and $P$ is a projection onto $\mathcal{S}^{\circ}=\{0\}$. Thus, $P=0$ and $Q=E$.

On the other hand, if $\mathcal{S}^{\circ} \neq\{0\}$ then, as a consequence of Theorem 6.2 and Proposition 5.6, there are infinitely many $J$-normal projections onto $\mathcal{S}$.

By Proposition 4.1, for a fixed pseudo-regular subspace $\mathcal{S}$ of $\mathcal{H}$, if $\mathcal{S}^{\circ}$ is the isotropic part of $\mathcal{S}$ and $\mathcal{M}$ is a subspace of $\mathcal{S}$ such that $\mathcal{S}=\mathcal{S}^{\circ}[\dot{+}] \mathcal{M}$ (i.e. $\mathcal{M}$ is a complement of $\mathcal{S}^{\circ}$ in $\mathcal{S}$ ), then $\mathcal{M}$ is a regular subspace of $\mathcal{H}$. Hence, consider

$$
\mathcal{Q}_{\mathcal{S}, \mathcal{M}}=\left\{Q \in \mathcal{Q}_{\mathcal{S}}: Q Q^{\#}=E_{\mathcal{M}}\right\},
$$

where $E_{\mathcal{M}}$ stands for the $J$-selfadjoint projection onto $\mathcal{M}$.

Notice that $\mathcal{Q}_{\mathcal{S}}$ can be written as the disjoint union of the family $\mathcal{Q}_{\mathcal{S}, \mathcal{M}}$, as $\mathcal{M}$ varies on the complements of $\mathcal{S}^{\circ}$ in $\mathcal{S}$ :

Lemma 6.4. If $\mathcal{S}$ is a pseudo-regular subspace of $\mathcal{H}$, then

$$
\mathcal{Q}_{\mathcal{S}}=\bigcup_{\left\{\mathcal{M}: \mathcal{S}=\mathcal{S}^{\circ}[+] \mathcal{M}\right\}} \mathcal{Q}_{\mathcal{S}, \mathcal{M}}
$$

where $\dot{U}$ denotes a disjoint union.

Proof. It is obvious that $\mathcal{Q}_{\mathcal{S}}=\bigcup_{\left\{\mathcal{M}: \mathcal{S}=\mathcal{S}^{\circ}[+] \mathcal{M}\right\}} \mathcal{Q}_{\mathcal{S}, \mathcal{M}}$. Suppose that $Q \in$ $\mathcal{Q}_{\mathcal{S}, \mathcal{M}_{1}} \cap \mathcal{Q}_{\mathcal{S}, \mathcal{M}_{2}}$, where $\mathcal{M}_{1}$ and $\mathcal{M}_{2}$ are regular subspaces of $\mathcal{H}$. Then,

$$
E_{\mathcal{M}_{1}}=Q Q^{\#}=E_{\mathcal{M}_{2}},
$$

or equivalently, $\mathcal{M}_{1}=\mathcal{M}_{2}$. Hence, $\mathcal{Q}_{\mathcal{S}, \mathcal{M}_{1}}=\mathcal{Q}_{\mathcal{S}, \mathcal{M}_{2}}$. 


\section{Parametrizing the deck $\mathcal{Q}_{\mathcal{S}, \mathcal{M}}$ for a pseudo-regular subspace $\mathcal{S}$}

The following paragraphs are devoted to studying those $J$-normal projections onto $\mathcal{S}$ which have a fixed regular part. Along this section operators are treated as block-operator matrices according to the orthogonal decomposition

$$
\mathcal{H}=\mathcal{S}^{\circ} \oplus\left(\mathcal{S} \ominus \mathcal{S}^{\circ}\right) \oplus \mathcal{S}^{\perp},
$$

and $P_{\mathcal{S}^{\perp}}, P_{\mathcal{S}^{\circ}}$ and $P_{\mathcal{S} \ominus \mathcal{S}^{\circ}}$ denote the orthogonal projections onto $\mathcal{S}^{\perp}, \mathcal{S}^{\circ}$ and $\mathcal{S} \ominus \mathcal{S}^{\circ}$, respectively.

If $\mathcal{M}$ is a regular subspace of $\mathcal{H}$ such that $\mathcal{S}=\mathcal{S}^{\circ}[\dot{+}] \mathcal{M}$, it is necessary to describe the fundamental symmetry $J$ and the $J$-selfadjoint projection $E_{\mathcal{M}}$ onto $\mathcal{M}$ as block-operator matrices.

Lemma 6.5. If $\mathcal{S}$ is a pseudo-regular subspace of $\mathcal{H}$, then $J$ is represented as the block-operator matrix

$$
J=\left(\begin{array}{ccc}
0 & 0 & a \\
0 & b & c \\
a^{*} & c^{*} & d
\end{array}\right) \begin{aligned}
& \mathcal{S}^{\circ} \\
& \mathcal{S} \ominus \mathcal{S}^{\circ}, \\
& \mathcal{S}^{\perp}
\end{aligned}
$$

where $a \in L\left(\mathcal{S}^{\perp}, \mathcal{S}^{\circ}\right), b=b^{*} \in G L\left(\mathcal{S} \ominus \mathcal{S}^{\circ}\right), c \in L\left(\mathcal{S}^{\perp}, \mathcal{S} \ominus \mathcal{S}^{\circ}\right)$ and $d=d^{*} \in$ $L\left(\mathcal{S}^{\perp}\right)$ satisfy the following equations:

$$
\left\{\begin{array}{rl}
a a^{*} & =I_{\mathcal{S}^{\circ}} \\
b^{2}+c c^{*} & =I_{\mathcal{S} \ominus \mathcal{S}^{\circ}} \\
a^{*} a+c^{*} c+d^{2} & =I_{\mathcal{S}^{\perp}} \\
b c+c d=a d=a c^{*} & =0
\end{array} .\right.
$$

Proof. Notice that $P_{\mathcal{S}^{\circ}} J P_{\mathcal{S}^{\circ}}=0$ because $\mathcal{S}^{\circ}$ is $J$-neutral. Also, $P_{\mathcal{S}^{\circ}} J P_{\mathcal{S} \ominus \mathcal{S}^{\circ}}=0$ because $\mathcal{S} \ominus \mathcal{S}^{\circ} \subseteq \mathcal{S}$ and $\mathcal{S}^{\circ} \subseteq \mathcal{S}^{[\perp]}$. Then,

$$
J=\left(\begin{array}{ccc}
0 & 0 & a \\
0 & b & c \\
a^{*} & c^{*} & d
\end{array}\right) .
$$

On the other hand, the system of equations (6.3) follows from $J^{2}=I$.

By Proposition 4.1, $\mathcal{S} \ominus \mathcal{S}^{\circ}$ is a regular subspace of $\mathcal{H}$. Furthermore, the regularity of $\mathcal{S} \ominus \mathcal{S}^{\circ}$ is equivalent to the range inclusion

$$
R(c) \subseteq R(b),
$$

see [7, Prop. 3.3]. Then, the second equation in (6.3) implies that $\mathcal{S} \ominus \mathcal{S}^{\circ} \subseteq R(b)$. Hence, $b$ is an invertible selfadjoint operator in $L\left(\mathcal{S} \ominus \mathcal{S}^{\circ}\right)$.

Remark 6.6. Observe that the operator $a \in L\left(\mathcal{S}^{\perp}, \mathcal{S}^{\circ}\right)$ appearing in the above lemma is a coisometry. Then, $a^{*} \in L\left(\mathcal{S}^{\circ}, \mathcal{S}^{\perp}\right)$ is a partial isometry with final space $J\left(\mathcal{S}^{\circ}\right)$. 
Indeed, by the block-operator matrix representation of $J$ given in (6.2), it is easy to see that $R\left(a^{*}\right)=J\left(\mathcal{S}^{\circ}\right)$. Hence,

$$
R\left(a^{*} a\right)=R\left(a^{*}\right)=J\left(\mathcal{S}^{\circ}\right) .
$$

Thus, $a^{*} a \in L\left(\mathcal{S}^{\perp}\right)$ is the orthogonal projection onto $J\left(\mathcal{S}^{\circ}\right)$.

The following lemma presents a block-matrix representation for the $J$-selfadjoint projection $E_{\mathcal{M}}$ onto a particular complement $\mathcal{M}$ of $\mathcal{S}^{\circ}$ in $\mathcal{S}$. This is a technical tool necessary to parametrize the $\operatorname{deck} \mathcal{Q}_{\mathcal{S}, \mathcal{M}}$.

Lemma 6.7. Given a pseudo-regular subspace $\mathcal{S}$ of $\mathcal{H}$, let $\mathcal{M}$ be a complement of $\mathcal{S}^{\circ}$ in $\mathcal{S}$. Then, the $J$-selfadjoint projection onto $\mathcal{M}$ is

$$
E_{\mathcal{M}}=\left(\begin{array}{ccc}
0 & a r^{*} b & a r^{*}(c+b r) \\
0 & I & b^{-1} c+r \\
0 & 0 & 0
\end{array}\right),
$$

where $r=\left.P_{\mathcal{S} \ominus \mathcal{S}^{\circ}} E_{\mathcal{M}} P_{J\left(\mathcal{S}^{\circ}\right)}\right|_{\mathcal{S}^{\perp}} \in L\left(\mathcal{S}^{\perp}, \mathcal{S} \ominus \mathcal{S}^{\circ}\right)$.

Proof. Suppose that $\mathcal{S}$ is a pseudo-regular subspace of $\mathcal{H}$. Then, by Proposition 4.1, $\mathcal{M}$ is regular.

Denote by $E_{\mathcal{M}}$ the $J$-selfadjoint projection onto $\mathcal{M}$. Since $R\left(E_{\mathcal{M}}\right)=\mathcal{M} \subseteq \mathcal{S}$ it follows that $P_{\mathcal{S}} \perp E_{\mathcal{M}}=0$, so that the third row in the matrix representation of $E_{\mathcal{M}}$ is zero. Also, since $\mathcal{S}^{\circ} \subseteq \mathcal{S}^{[\perp]} \subseteq \mathcal{M}^{[\perp]}=N\left(E_{\mathcal{M}}\right)$, it follows that $E_{\mathcal{M}} P_{\mathcal{S}^{\circ}}=0$. So that the first column is also zero. Therefore,

$$
E_{\mathcal{M}}=\left(\begin{array}{lll}
0 & u & v \\
0 & p & q \\
0 & 0 & 0
\end{array}\right)
$$

where $u \in L\left(\mathcal{S} \ominus \mathcal{S}^{\circ}, \mathcal{S}^{\circ}\right), v \in L\left(\mathcal{S}^{\perp}, \mathcal{S}^{\circ}\right), p \in L\left(\mathcal{S} \ominus \mathcal{S}^{\circ}\right)$ and $q \in L\left(\mathcal{S}^{\perp}, \mathcal{S} \ominus \mathcal{S}^{\circ}\right)$ satisfy

$$
\left\{\begin{array}{l}
u p=u \\
u q=v \\
p^{2}=p \\
p q=q
\end{array} .\right.
$$

Thus, $p=\left.P_{\mathcal{S} \ominus \mathcal{S}^{\circ}} E_{\mathcal{M}}\right|_{\mathcal{S} \ominus \mathcal{S}} \circ$ is a projection with

$R(p)=P_{\mathcal{S} \ominus \mathcal{S}^{\circ}} E_{\mathcal{M}}\left(\mathcal{S} \ominus \mathcal{S}^{\circ}\right)=P_{\mathcal{S} \ominus \mathcal{S}^{\circ}} E_{\mathcal{M}}(\mathcal{S})=P_{\mathcal{S} \ominus \mathcal{S}^{\circ}}(\mathcal{M})=P_{\mathcal{S} \ominus \mathcal{S}^{\circ}}(\mathcal{S})=\mathcal{S} \ominus \mathcal{S}^{\circ}$, because $\mathcal{S}^{\circ} \subseteq N\left(P_{\mathcal{S} \ominus \mathcal{S}^{\circ}}\right) \cap N\left(E_{\mathcal{M}}\right)$. Hence, $p=I_{\mathcal{S} \ominus \mathcal{S}^{\circ}}$.

Furthermore, $E_{\mathcal{M}}$ is $J$-selfadjoint if and only if

$$
J E_{\mathcal{M}}=\left(\begin{array}{ccc}
0 & 0 & 0 \\
0 & b & b q \\
0 & a^{*} u+c^{*} & \left(a^{*} u+c^{*}\right) q
\end{array}\right)
$$

is selfadjoint, or equivalently, if

$$
a^{*} u+c^{*}=q^{*} b
$$


By (6.3), $a a^{*}=I_{\mathcal{S}^{\circ}}$ and $a c^{*}=0$. Thus, multiplying on the left by $a$, it follows that $u=a q^{*} b$. Thus,

$$
E_{\mathcal{M}}=\left(\begin{array}{ccc}
0 & a q^{*} b & a q^{*} b q \\
0 & I & q \\
0 & 0 & 0
\end{array}\right)
$$

where $q=\left.P_{\mathcal{S} \ominus \mathcal{S}^{\circ}} E_{\mathcal{M}}\right|_{\mathcal{S}^{\perp}}$. Replacing $u$ is (6.6), notice that $q$ satisfies $a^{*} a q^{*} b+$ $c^{*}=q^{*} b$, or equivalently,

$$
q=q\left(a^{*} a\right)+b^{-1} c .
$$

Therefore, if $r=q\left(a^{*} a\right)$ then $a q^{*} b=a\left(c^{*} b^{-1}+r^{*}\right) b=a r^{*} b$, and (6.5) follows.

Finally, a block-matrix representation of a projection $Q \in L(\mathcal{H})$ onto $\mathcal{S}$ is needed. Since $R(Q)=\mathcal{S}$, observe that $P_{\mathcal{S} \circ} Q P_{\mathcal{S}} \circ=P_{\mathcal{S}^{\circ}}, P_{\mathcal{S} \ominus \mathcal{S}^{\circ}} Q P_{\mathcal{S} \ominus \mathcal{S}^{\circ}}=$ $P_{\mathcal{S} \ominus \mathcal{S}^{\circ}}$ and

$$
P_{\mathcal{S}} \circ Q P_{\mathcal{S} \ominus \mathcal{S}^{\circ}}=P_{\mathcal{S} \ominus \mathcal{S}^{\circ}} Q P_{\mathcal{S}} \circ=0 .
$$

Then, $Q$ is represented as the block-operator matrix

$$
Q=\left(\begin{array}{lll}
I & 0 & x \\
0 & I & y \\
0 & 0 & 0
\end{array}\right)
$$

where $x=\left.P_{\mathcal{S}^{\circ}} Q\right|_{\mathcal{S}^{\perp}} \in L\left(\mathcal{S}^{\perp}, \mathcal{S}^{\circ}\right)$ and $y=\left.P_{\mathcal{S} \ominus \mathcal{S}^{\circ}} Q\right|_{\mathcal{S}^{\perp}} \in L\left(\mathcal{S}^{\perp}, \mathcal{S} \ominus \mathcal{S}^{\circ}\right)$.

Furthermore, if $Q \in \mathcal{Q}_{\mathcal{S}, \mathcal{M}}$ then, by Theorem 3.1, $P=Q-E_{\mathcal{M}}$ is a projection onto $\mathcal{S}^{\circ}$ such that $P P^{\#}=P^{\#} P=0$. Moreover, by (6.5), $P$ has the form

$$
P=Q-E_{\mathcal{M}}=\left(\begin{array}{ccc}
I & -a r^{*} b & x-a r^{*}(c+b r) \\
0 & 0 & y-b^{-1} c-r \\
0 & 0 & 0
\end{array}\right) .
$$

But, $R(P)=\mathcal{S}^{\circ}$ if and only if

$$
y=b^{-1} c+r .
$$

Also, $P P^{\#}=0$ if and only if $P J P^{*}=0$, or equivalently,

$$
\left(\begin{array}{ccc}
I & -a r^{*} b & z \\
0 & 0 & 0 \\
0 & 0 & 0
\end{array}\right)\left(\begin{array}{ccc}
0 & 0 & a \\
0 & b & c \\
a^{*} & c^{*} & d
\end{array}\right)\left(\begin{array}{ccc}
I & 0 & 0 \\
-b r a^{*} & 0 & 0 \\
z^{*} & 0 & 0
\end{array}\right)=0,
$$

where $z=x-a r^{*}(c+b r)$. But the above equation is equivalent to

$$
z\left(I-r^{*} b c\right)^{*} a^{*}+a\left(I-r^{*} b c\right) z^{*}+z d z^{*}+a r^{*} b^{3} r a^{*}=0 .
$$

The following lemma is devoted to describe the solutions of (6.8), where $a$, $b, c, d$ and $r$ are the operators appearing in (6.2) and in (6.5). 
Lemma 6.8. An operator $z \in L\left(\mathcal{S}^{\perp}, \mathcal{S}^{\circ}\right)$ is a solution of (6.8) if and only if there exist $A=-A^{*} \in L\left(\mathcal{S}^{\circ}\right)$ and $B \in L\left(\mathcal{S}^{\perp}, \mathcal{S}^{\circ}\right)$ with $J\left(\mathcal{S}^{\circ}\right) \subseteq N(B)$ such that

$$
z=\left(A+\operatorname{Re}\left(B c^{*} b r a^{*}\right)-\frac{1}{2}\left(B d B^{*}+a r^{*} b^{3} r a^{*}\right)\right) a+B .
$$

Proof. Let $z \in L\left(\mathcal{S}^{\perp}, \mathcal{S}^{\circ}\right)$ be a solution of (6.8) and consider the operators

$$
z_{1}=z\left(a^{*} a\right) \quad \text { and } \quad z_{2}=z\left(I_{\mathcal{S}^{\perp}}-a^{*} a\right) .
$$

Notice that $z_{1}\left(I-r^{*} b c\right)^{*} a^{*}+a\left(I-r^{*} b c\right) z_{1}^{*}=z_{1} a^{*}+a z_{1}^{*}=2 \operatorname{Re}\left(z_{1} a^{*}\right)$ because $a c^{*}=c a^{*}=0$. Also,

$$
z_{2}\left(I-r^{*} b c\right)^{*} a^{*}+a\left(I-r^{*} b c\right) z_{2}^{*}=-z_{2} c^{*} b r a^{*}-a r^{*} b c z_{2}^{*}=-2 \operatorname{Re}\left(z_{2} c^{*} b r a^{*}\right),
$$

because $z_{2} a^{*}=a z_{2}^{*}=0$. On the other hand, since $a d=d a^{*}=0$ it is easy to see that

$$
z d z^{*}=\left(z_{1}+z_{2}\right) d\left(z_{1}+z_{2}\right)^{*}=z_{2} d z_{2}^{*} .
$$

Therefore, (6.8) is equivalent to

$$
2 \operatorname{Re}\left(z_{1} a^{*}\right)=2 \operatorname{Re}\left(z_{2} c^{*} b r a^{*}\right)-z_{2} d z_{2}^{*}-a r^{*} b^{3} r a^{*} .
$$

Then, considering the antihermitian operator $A=i \operatorname{Im}\left(z_{1} a^{*}\right) \in L\left(\mathcal{S}^{\circ}\right)$, it follows that

$$
\begin{aligned}
z_{1} & =\left(z_{1} a^{*}\right) a=\left(i \operatorname{Im}\left(z_{1} a^{*}\right)+\operatorname{Re}\left(z_{1} a^{*}\right)\right) a \\
& =\left(A+\operatorname{Re}\left(z_{2} c^{*} b r a^{*}\right)-\frac{1}{2}\left(z_{2} d z_{2}^{*}+a r^{*} b^{3} r a^{*}\right)\right) a .
\end{aligned}
$$

Hence, $B=z_{2} \in L\left(\mathcal{S}^{\perp}, \mathcal{S}^{\circ}\right)$ satisfies $J\left(\mathcal{S}^{\circ}\right) \subseteq N(B)$ and

$$
z=z_{1}+z_{2}=\left(A+\operatorname{Re}\left(B c^{*} b r a^{*}\right)-\frac{1}{2}\left(B d B^{*}+a r^{*} b^{3} r a^{*}\right)\right) a+B .
$$

Conversely, given an antihermitian operator $A \in L\left(\mathcal{S}^{\circ}\right)$ and $B \in L\left(\mathcal{S}^{\perp}, \mathcal{S}^{\circ}\right)$ such that $N(b)^{\perp} \subseteq N(d)$, consider

$$
z_{A, B}:=\left(A+\operatorname{Re}\left(B c^{*} b r a^{*}\right)-\frac{1}{2}\left(B d B^{*}+a r^{*} b^{3} r a^{*}\right)\right) a+B .
$$

Then, it is easy to see that $z_{A, B} \in L\left(\mathcal{S}^{\perp}, \mathcal{S}^{\circ}\right)$ is a solution of (6.8).

Finally, it is possible to parametrize the $\operatorname{deck} \mathcal{Q}_{\mathcal{S}, \mathcal{M}}$ as follows:

Theorem 6.9. Let $Q \in L(\mathcal{H})$ be a projection onto a pseudo-regular subspace $\mathcal{S}$ of $\mathcal{H}$. Suppose that $\mathcal{M}$ is a regular subspace of $\mathcal{H}$ such that $\mathcal{S}=\mathcal{S}^{\circ}+\mathcal{M}$. Then, $Q \in \mathcal{Q}_{\mathcal{S}, \mathcal{M}}$ if and only if

$$
Q=\left(\begin{array}{ccc}
I & 0 & \left(A+\operatorname{Re}\left(B c^{*} b r a^{*}\right)-\frac{1}{2}\left(B d B^{*}+a r^{*} b^{3} r a^{*}\right)\right) a+B+a r^{*}(c+b r) \\
0 & I & b^{-1} c+r \\
0 & 0 & 0
\end{array}\right),
$$

where $r=P_{\mathcal{S} \ominus \mathcal{S}^{\circ}} E_{\mathcal{M}}\left(a^{*} a\right) \in L\left(\mathcal{S}^{\perp}, \mathcal{S} \ominus \mathcal{S}^{\circ}\right), A=-A^{*} \in L\left(\mathcal{S}^{\circ}\right)$ and $B \in$ $L\left(\mathcal{S}^{\perp}, \mathcal{S}^{\circ}\right)$ is such that $J\left(\mathcal{S}^{\circ}\right) \subseteq N(B)$. 
Proof. Suppose that $Q \in \mathcal{Q}_{\mathcal{S}, \mathcal{M}}$, i.e. $Q \in L(\mathcal{H})$ is a $J$-normal projection onto $\mathcal{S}$ satisfying $Q Q^{\#}=Q^{\#} Q=E_{\mathcal{M}}$. Then, $P=Q-E_{\mathcal{M}}$ is a projection onto $\mathcal{S}^{\circ}$ such that $P P^{\#}=P^{\#} P=0$. Hence, if $Q$ is written as in (6.7) it follows that $y=b^{-1} c$.

Then, by the discussion above,

$$
P=\left(\begin{array}{ccc}
I & -a r^{*} b & x-a r^{*}(c+b r) \\
0 & 0 & 0 \\
0 & 0 & 0
\end{array}\right)
$$

where $z=x-a r^{*}(c+b r)$ is a solution of (6.8). Thus, by Proposition 6.8, there exist $A=-A^{*} \in L\left(\mathcal{S}^{\circ}\right)$ and $B \in L\left(\mathcal{S}^{\perp}, \mathcal{S}^{\circ}\right)$ with $J\left(\mathcal{S}^{\circ}\right) \subseteq N(B)$ such that

$$
P=\left(\begin{array}{ccc}
I & -a r^{*} b & \left(A+\operatorname{Re}\left(B c^{*} b r a^{*}\right)-\frac{1}{2}\left(B d B^{*}+a r^{*} b^{3} r a^{*}\right)\right) a+B \\
0 & 0 & 0 \\
0 & 0 & 0
\end{array}\right) .
$$

Therefore,

$$
Q=\left(\begin{array}{ccc}
I & 0 & \left(A+\operatorname{Re}\left(B c^{*} b r a^{*}\right)-\frac{1}{2}\left(B d B^{*}+a r^{*} b^{3} r a^{*}\right)\right) a+B+a r^{*}(c+b r) \\
0 & I & b^{-1} c+r \\
0 & 0 & 0
\end{array}\right) .
$$

The converse follows immediately.

Given a pseudo regular subspace $\mathcal{S}$ of $\mathcal{H}$, denote by $\mathcal{C}\left(\mathcal{S}^{\circ}\right)$ the set of complements of $\mathcal{S}^{\circ}$ in $\mathcal{S}$. Recall that, by Lemma 6.4, the set of $J$-normal projections onto $\mathcal{S}$ is decomposed as

$$
\mathcal{Q}_{\mathcal{S}}=\bigcup_{\mathcal{M} \in \mathcal{C}\left(\mathcal{S}^{\circ}\right)} \mathcal{Q}_{\mathcal{S}, \mathcal{M}}
$$

Furthermore, for a fixed $\mathcal{M} \in \mathcal{C}\left(\mathcal{S}^{\circ}\right)$, Theorem 6.9 states that the deck $\mathcal{Q}_{\mathcal{S}, \mathcal{M}}$ is parametrized by the bijection $\Psi_{\mathcal{M}}: \mathcal{A H}\left(\mathcal{S}^{\circ}\right) \times \mathcal{N}_{\circ} \rightarrow \mathcal{Q}_{\mathcal{S}, \mathcal{M}}$ given by

$$
\Psi_{\mathcal{M}}(A, B)=\left(\begin{array}{ccc}
I & 0 & \left(A+\operatorname{Re}\left(B c^{*} b r a^{*}\right)-\frac{1}{2}\left(B d B^{*}+a r^{*} b^{3} r a^{*}\right)\right) a+B+a r^{*}(c+b r) \\
0 & I & b^{-1} c+r \\
0 & 0 & 0
\end{array}\right),
$$

where $\mathcal{A H}\left(\mathcal{S}^{\circ}\right)$ stands for the real vector space of antihermitian operators acting on $\mathcal{S}^{\circ}$ and $\mathcal{N}_{\circ}$ is the set composed by those operators $B \in L\left(\mathcal{S}^{\perp}, \mathcal{S}^{\circ}\right)$ such that $J\left(\mathcal{S}^{\circ}\right) \subseteq N(B)$.

Therefore, the set $\mathcal{Q}_{\mathcal{S}}$ of $J$-normal projections onto $\mathcal{S}$ is parametrized as follows:

Theorem 6.10. Let $\mathcal{S}$ be a pseudo-regular subspace of $\mathcal{H}$. Then, the function $\Psi: \mathcal{R C}\left(\mathcal{S}^{\circ}\right) \times \mathcal{A H}\left(\mathcal{S}^{\circ}\right) \times \mathcal{N}_{\circ} \rightarrow \mathcal{Q}_{\mathcal{S}}$ defined by

$$
\Psi(\mathcal{M}, A, B)=\left(\begin{array}{ccc}
I & 0 & \left(A+\operatorname{Re}\left(B c^{*} b r a^{*}\right)-\frac{1}{2}\left(B d B^{*}+a r^{*} b^{3} r a^{*}\right)\right) a+B+a r^{*}(c+b r) \\
0 & I & b^{-1} c+r \\
0 & 0 & 0
\end{array}\right),
$$

is one-to one. 
Observe that in the expression defining $\Psi$ appears the operator

$$
r=\left.P_{\mathcal{S} \ominus \mathcal{S}^{\circ}} E_{\mathcal{M}} P_{J\left(\mathcal{S}^{\circ}\right)}\right|_{\mathcal{S}^{\perp}} \in L\left(\mathcal{S}^{\perp}, \mathcal{S} \ominus \mathcal{S}^{\circ}\right),
$$

given in Lemma 6.7, where $P_{\mathcal{S} \ominus \mathcal{S}^{\circ}}$ and $P_{J\left(\mathcal{S}^{\circ}\right)}$ are the orthogonal projections onto $\mathcal{S} \ominus \mathcal{S}^{\circ}$ and $J\left(\mathcal{S}^{\circ}\right)$, respectively, and $E_{\mathcal{M}}$ is the $J$-selfadjoint projection onto $\mathcal{M}$.

\section{An interesting particular deck: $\mathcal{Q}_{\mathcal{S}, \mathcal{S} \ominus \mathcal{S}^{\circ}}$}

Let $\mathcal{S}$ be a fixed pseudo-regular subspace of a Krein space $\mathcal{H}$ with fundametal symmetry $J$. These paragraphs are devoted to describe the set $\mathcal{Q}_{\mathcal{S}, \mathcal{S} \ominus \mathcal{S}^{\circ}}$, i.e. the family of $J$-normal projections $Q \in L(\mathcal{H})$ onto $\mathcal{S}$ such that $Q Q^{\#}$ is the $J$-selfadjoint projection onto the (regular) subspace $\mathcal{S} \ominus \mathcal{S}^{\circ}$. In this particular deck there is a minimal norm projection, see Remark 6.12.

First of all, since $\mathcal{S} \ominus \mathcal{S}^{\circ}$ is a complement of $\mathcal{S}^{\circ}$ in $\mathcal{S}$, it follows by Lemma 6.7 that the $J$-selfadjoint projection onto $\mathcal{S} \ominus \mathcal{S}^{\circ}$ (hereafter denoted by $E$ ) is the block-operator matrix given by (6.5), where

$$
r=\left.P_{\mathcal{S} \ominus \mathcal{S}^{\circ}} E P_{J\left(\mathcal{S}^{\circ}\right)}\right|_{\mathcal{S}^{\perp}} \in L\left(\mathcal{S}^{\perp}, \mathcal{S} \ominus \mathcal{S}^{\circ}\right) .
$$

But, $J\left(\mathcal{S}^{\circ}\right) \subseteq J\left(\mathcal{S}^{\circ}\right)+\mathcal{S}^{[\perp]}=J\left(\mathcal{S}^{\circ}+\mathcal{S}^{\perp}\right)=J\left(\left(\mathcal{S} \ominus \mathcal{S}^{\circ}\right)^{\perp}\right)=N(E)$. Therefore, $r=0$ and the block-operator matrix representation of $E$ is

$$
E=\left(\begin{array}{ccc}
0 & 0 & 0 \\
0 & I & b^{-1} c \\
0 & 0 & 0
\end{array}\right)
$$

Furthermore, as a consequence of Theorem $6.9, \mathcal{Q}_{\mathcal{S}, \mathcal{S} \ominus \mathcal{S}^{\circ}}$ is parametrized as:

Proposition 6.11. Let $\mathcal{S}$ be a pseudo-regular subspace of a Krein space $\mathcal{H}$ with fundametal symmetry $J$. A projection $Q$ onto $\mathcal{S}$ satisfies $Q Q^{\#}=Q^{\#} Q=E$ if and only if

$$
Q=\left(\begin{array}{ccc}
I & 0 & \left(A-\frac{1}{2} B d B^{*}\right) a+B \\
0 & I & b^{-1} c \\
0 & 0 & 0
\end{array}\right),
$$

where $a, b, c$ and $d$ are the operators appearing in (6.2), $A=-A^{*} \in L\left(\mathcal{S}^{\circ}\right)$ and $B \in L\left(\mathcal{S}^{\perp}, \mathcal{S}^{\circ}\right)$ is such that $J\left(\mathcal{S}^{\circ}\right) \subseteq N(B)$.

Remark 6.12. In this particular case it is possible to estimate

$$
\min \left\{\|Q\|: Q \in \mathcal{Q}_{\mathcal{S}, \mathcal{S} \ominus \mathcal{S}^{\circ}}\right\} .
$$

Indeed, if $P_{0}$ is the orthogonal projection onto $\mathcal{S}^{\circ}$ and $E$ stands for the $J$ selfadjoint projection onto $\mathcal{S} \ominus \mathcal{S}^{\circ}$, then $Q_{0}=E+P_{0} \in \mathcal{Q}_{\mathcal{S}, \mathcal{S} \ominus \mathcal{S}^{\circ}}$. Furthermore,

$$
\left\|Q_{0}\right\|^{2}=\left\|Q_{0} Q_{0}^{*}\right\|=\left\|E E^{*}+P_{0}\right\|=\max \left\{\left\|E E^{*}\right\|,\left\|P_{0}\right\|\right\}=\left\|E E^{*}\right\|=\|E\|^{2},
$$


because $R\left(E E^{*}\right)=\mathcal{S} \ominus \mathcal{S}^{\circ}$ is orthogonal to $R\left(P_{0}\right)=\mathcal{S}^{\circ}$. Therefore, $\left\|Q_{0}\right\|=\|E\|$.

On the other hand, if $Q \in \mathcal{Q}_{\mathcal{S}, \mathcal{S} \ominus \mathcal{S}^{\circ}}$ then there exists a (unique) $P=P^{2} \in$ $L(\mathcal{H})$ such that $P P^{\#}=P^{\#} P=0$ and $Q=E+P$.

Consider a sequence $\left\{x_{n}\right\}_{n \geq 1}$ in the unit ball of $\mathcal{H}$ such that $\left\|E x_{n}\right\| \rightarrow\|E\|$ as $n \rightarrow \infty$. Then,

$$
\|Q\|^{2} \geq\left\|Q x_{n}\right\|^{2}=\left\|E x_{n}\right\|^{2}+\left\|P x_{n}\right\|^{2} \geq\left\|E x_{n}\right\|^{2} \rightarrow\|E\|^{2}=\left\|Q_{0}\right\|^{2} .
$$

Hence, $\left\|Q_{0}\right\|=\min \left\{\|Q\|: Q \in \mathcal{Q}_{\mathcal{S}, \mathcal{S} \ominus \mathcal{S}^{\circ}}\right\}$.

\section{Acknowledgement}

The authors are indebted to Prof. Tsuyoshi Ando not only for several discussions on the material of this manuscript, but also for allowing the inclusion of the highlighted material.

The authors would also like to thank the anonymous referee for pointing out several typos and for some suggestions that improved the exposition of the paper.

\section{References}

[1] T. Ando, Linear operators on Krein spaces, Hokkaido University, Sapporo, Japan, 1979.

[2] T. Ando, Unbounded or bounded idempotent operators in Hilbert space, Linear Algebra Appl. (2011), doi:10.1016/j.laa.2011.06.047.

[3] T. Ando, Projections in Krein spaces, Linear Algebra Appl. 431 (2009), no. $12,2346-2358$.

[4] J. A. Ball and J. W. Helton, Factorization Results Related to Shifts in an Indefinite Metric, Integ. Equ. Oper. Theory 5 (1982), 632-658.

[5] J. A. Ball and J. W. Helton, A Beurling-Lax theorem for the Lie group $U(m, n)$ which contains most classical interpolation theory, J. Operator Theory 7 (1982), 179-189.

[6] J. Bognar, Indefinite inner product spaces, Springer-Verlag, BerlinHeidelberg-New York, 1974.

[7] G. Corach, A. Maestripieri and D. Stojanoff, Oblique projections and Schur complements, Acta Sci. Math. (Szeged) 67 (2001), no. 1-2, 337356.

[8] F. Deutsch, The angle between subspaces of a Hilbert space, Approximation theory, wavelets and applications (Maratea, 1994), 107-130, NATO Adv. Sci. Inst. Ser. C Math. Phys. Sci., 454, Kluwer Acad. Publ., Dordrecht, 1995. 
[9] A. Gheondea, On the geometry of pseudo-regular subspaces of a Krein space, in Spectral theory of linear operators and related topics, Birkhäuser Verlag, 1984, 141-156.

[10] A. Gheondea, Pseudo-regular spectral functions in Krein spaces, J. Operator Theory 12 (1984), 349-358.

[11] A. Gheondea and P. Jonas, A characterization of spectral functions of definitizable operators, J. Operator Theory 17 (1987), 99-119.

[12] I. S. Iokhvidov and T. Ya. Azizov, Linear Operators in spaces with an indefinite metric, John Wiley and sons, 1989.

[13] P. Jonas, On spectral distributions of definitizable operators in Krein spaces, in Spectral theory, Banach Center Publications 6 (1982), 301-311.

[14] V. Strauss, On models of function type for a special class of normal operators in Krein spaces and their polar representation, Methods of Functional Analysis and Topology 13 (2007), no. 1, 67-82.

\author{
Alejandra Maestripieri \\ Facultad de Ingeniería - Universidad de Buenos Aires \\ and \\ Instituto Argentino de Matemática "Alberto P. Calderón" \\ amaestri@ing.uba.ar
}

\title{
Francisco Martínez Pería
}

Facultad de Ciencias Exactas - Universidad Nacional de La Plata

and

Instituto Argentino de Matemática "Alberto P. Calderón"

Saavedra 15, Piso 3

(1083) Buenos Aires

Argentina

francisco@mate.unlp.edu.ar 